\title{
ON SUBADDITIVE DUALITY FOR CONIC MIXED-INTEGER PROGRAMS*
}

\author{
BURAK KOCUK ${ }^{\dagger}$ AND DIEGO A. MORÁN R. ${ }^{\ddagger}$
}

\begin{abstract}
In this paper, we show that the subadditive dual of a feasible conic mixed-integer program (MIP) is a strong dual whenever it is feasible. Moreover, we show that this dual feasibility condition is equivalent to feasibility of the conic dual of the continuous relaxation of the conic MIP. In addition, we prove that all known conditions and other "natural" conditions for strong duality, such as strict mixed-integer feasibility, boundedness of the feasible set, or essentially strict feasibility, imply that the subadditive dual is feasible. As an intermediate result, we extend the so-called "finiteness property" from full-dimensional convex sets to intersections of full-dimensional convex sets and Dirichlet convex sets.
\end{abstract}

Key words. mixed-integer programming, conic programming, subadditive duality

AMS subject classifications. 90C11, 90C46

DOI. $10.1137 / 18 \mathrm{M} 1210812$

\section{Introduction.}

Duality for mixed-integer programs (MIPs). Duality is an important concept in mathematical programming for both analyzing the properties of optimization problems and constructing solution methods. For an optimization problem in minimization (resp., maximization) form, a dual problem is called weak if its optimal value provides a lower (resp., upper) bound to the optimal value of the primal problem. Furthermore, a dual problem is called strong if (i) there is zero duality gap, that is, its optimal value is equal to that of the primal problem, and (ii) it is solvable, that is, the optimal value is attained. The first property ensures that the dual problem is giving the best possible bound and the second property provides a way to obtain this best bound by finding an optimal dual feasible solution. Both of these properties are crucial in the development of effective optimization algorithms.

It is well known that linear programming (LP) and conic programming $(\mathrm{CP})$ problems and their respective duals satisfy strong duality under mild conditions (such as boundedness and feasibility or strict feasibility) [6]. The case of mixed-integer linear programs (MILP) is more involved and requires the definition of a functional dual problem, the so-called subadditive dual, which is a strong dual when the data defining the primal problem is rational $[15,21]$. Recently, this latter duality result was extended to conic MIPs in [20] under a mixed-integer strict feasibility requirement, similar to the one needed in the continuous conic case. We also note that other types of duals have been studied in the case of general mixed-integer nonlinear programming (MINLP) problems. For instance, the Karush-Kuhn-Tucker (KKT) optimality conditions are generalized for MINLPs in [3], Lagrangian-based methods are used

${ }^{*}$ Received by the editors August 30, 2018; accepted for publication (in revised form) June 11, 2019; published electronically September 19, 2019.

https://doi.org/10.1137/18M1210812

Funding: The work of the authors was supported by Conicyt Fondecyt grant 11161085.

†Industrial Engineering Program, Sabancı University, Istanbul 34956, Turkey (burakkocuk@ sabanciuniv.edu).

${ }^{\ddagger}$ School of Business, Universidad Adolfo Ibáñez, Santiago 7941169, Chile (diego.moran@uai.cl). 
in $[10,14]$, and other geometric [4] or algebraic [27] approaches are utilized to obtain strong duality results in particular cases.

Conic MIPs. Conic MIP problems generalize MILPs and have significantly more expressive power in terms of modeling. To name just a few application areas, conic MIPs are used in options pricing [22], power distribution systems [17], Euclidean $k$ center problems [8], and engineering design [12]. We note here that all the conic MIPs used in these applications include binary variables, and that this feature, rather than being the exception, is a general rule when modeling real-life problems.

In spite of the growing interest in conic MIP applications, conic MIP solvers are not as mature as their MILP counterparts. Although the subadditive dual for linear/conic MIPs do not yield straightforward solution procedures, any dual feasible solution generates a valid inequality for the primal problem. Moreover, if strong duality holds, all cutting planes are equal to or dominated by a cutting plane obtained from such a solution $[30,20]$. We know that these valid inequalities are extremely useful for MILPs (see, for instance, [7]) and one may expect that cutting planes designed for conic MIPs will help solve them more efficiently. Recent work on cutting planes for conic MIPs includes generalizations of Gomory cuts [9], rounding cuts $[1,26]$, split/disjunctive cuts [25], and minimal valid inequalities [16].

Our contributions. In this paper, we study sufficient conditions under which the subadditive dual for conic MIPs is a strong dual. Although the mixed-integer strict feasibility condition in [20] is somewhat reasonable, it has two main drawbacks: (i) it may not always hold in practical problem settings (for instance, if the conic MIP includes binary variables or its feasible region is not full-dimensional), and (ii) it is not "easy" to check in general, that is, there is no known polynomial time algorithm for this task. This motivates the search for other sufficient conditions.

Our main result is the following sufficient condition for strong duality: if the primal conic MIP and the subadditive dual problems are both feasible, then strong duality holds. Furthermore, under the assumption that the primal problem is feasible, we show that feasibility of the subadditive dual is equivalent to feasibility of the conic dual of the continuous relaxation of the primal.

Moreover, we prove that under some "natural" conditions, which include all known sufficient conditions for strong duality in the linear and conic MIP cases, the conic dual of the continuous relaxation of the conic MIP is feasible. As a consequence of our main result, we obtain that these "natural" conditions, some of which include cases that are "easy" to check, imply that the subadditive dual is a strong dual.

Finally, as an intermediate result of independent interest, we find a sufficient condition for the finiteness of the objective function of the convex MIP implying the finiteness of the objective function of its continuous relaxation. This is an extension of the "finiteness property" result given in [18] for rational polyhedra and in [20] for full-dimensional convex sets to intersections of full-dimensional convex sets and Dirichlet convex sets (a class of sets first studied in [19]).

Organization of the rest of the paper. In section 2, we review some results from the literature and give precise statements of our main contributions. The proof of the new sufficient condition for strong duality is presented in section 3 . In section 4 , we study properties of Dirichlet convex sets and give a proof of our extension of the finiteness property. In section 5 , we prove that some generally occurring conditions on the primal conic MIP imply dual feasibility. Finally, some concluding remarks are discussed in section 6 . 


\section{Main results.}

2.1. Preliminaries. For a set $X \subseteq \mathbb{R}^{n}$, we denote its interior as $\operatorname{int}(X)$, its recession cone as rec.cone $(X)$, its affine hull as aff $(X)$, its (affine) dimension as $\operatorname{dim}(X)$, and its convex hull as $\operatorname{conv}(X)$. We next review some definitions that are necessary to formulate a conic MIP.

Definition 2.1 (regular cone). A cone $K \subseteq \mathbb{R}^{m}$ is called regular if it is closed, convex, pointed, and full-dimensional.

Definition 2.2 (conic inequality). A conic inequality with respect to a regular cone $K$ is defined as $x \succeq_{K} y$, meaning that $x-y \in K$. We will denote the relation $x \in \operatorname{int}(K)$ alternatively by $x \succ_{K} 0$.

We define a generic conic MIP as follows:

$$
\begin{aligned}
z^{*}:=\inf & c^{T} x+d^{T} y \\
\text { s.t. } & A x+G y \succeq_{K} b, \\
& x \in \mathbb{Z}^{n_{1}}, y \in \mathbb{R}^{n_{2}},
\end{aligned}
$$

where $K \subseteq \mathbb{R}^{m}$ is a regular cone, $c \in \mathbb{R}^{n_{1}}, d \in \mathbb{R}^{n_{2}}, A \in \mathbb{R}^{m \times n_{1}}, G \in \mathbb{R}^{m \times n_{2}}$, and $b \in \mathbb{R}^{m}$.

The following definitions are instrumental in the description of the subadditive dual problem of (1).

Definition 2.3 (subadditive function). A function $f: \mathbb{R}^{m} \rightarrow \mathbb{R}$ is subadditive if $f(u+v) \leq f(u)+f(v)$ for all $u, v \in \mathbb{R}^{m}$.

Definition 2.4 (nondecreasing function). A function $f: \mathbb{R}^{m} \rightarrow \mathbb{R}$ is nondecreasing with respect to a regular cone $K \subseteq \mathbb{R}^{m}$ if $u \succeq_{K} v \Rightarrow f(u) \geq f(v)$.

We denote the set of subadditive functions that are nondecreasing with respect to a regular cone $K \subseteq \mathbb{R}^{m}$ by $\mathcal{F}_{K}$ and for $f \in \mathcal{F}_{K}$ we define $\bar{f}(x):=\lim \sup _{\delta \rightarrow 0^{+}} \frac{f(\delta x)}{\delta}$.

The subadditive dual problem of (1) is

$$
\begin{aligned}
\rho^{*}:=\sup & f(b) \\
\text { s.t. } & f\left(A^{j}\right)=-f\left(-A^{j}\right)=c_{j}, \quad j=1, \ldots, n_{1}, \\
& \bar{f}\left(G^{j}\right)=-\bar{f}\left(-G^{j}\right)=d_{j}, \quad j=1, \ldots, n_{2}, \\
& f(0)=0, \\
& f \in \mathcal{F}_{K},
\end{aligned}
$$

where $A^{j}$ (resp., $G^{j}$ ) denotes the $j$ th column of the matrix $A$ (resp., $G$ ).

It is not hard to show that the subadditive dual (2) is a weak dual to the primal conic MIP (1), that is, any dual feasible solution provides a lower bound for the optimal value of the primal (see, for instance, Proposition 3.2 in [20]). The following result provides a sufficient condition for the subadditive dual to be a strong dual for (1), that is, there is zero duality gap (i.e., $z^{*}=\rho^{*}$ ) and the subadditive dual is solvable (i.e., there exists a function $f$ feasible for the dual such that $f(b)=\rho^{*}$ ).

Theorem 2.5 (Theorem 2.4 of [20]). If $z^{*}>-\infty$ and there exists $(\hat{x}, \hat{y}) \in$ $\mathbb{Z}^{n_{1}} \times \mathbb{R}^{n_{2}}$ such that $A \hat{x}+G \hat{y} \succ_{K} b$, then the dual problem (2) is a strong dual for (1).

We note here that the sufficient condition in Theorem 2.5 is similar to the strict feasibility condition for strong duality in the conic case (see condition (b) in Theorem 2.6 with $A_{2}=0, b_{2}=0$ ). 
Theorem 2.6 (duality for conic programming $[29,6]$ ). Let $A_{1} \in \mathbb{R}^{m_{1} \times n}, A_{2} \in$ $\mathbb{R}^{m_{2} \times n}, c \in \mathbb{R}^{n}, b_{1} \in \mathbb{R}^{m_{1}}, b_{2} \in \mathbb{R}^{m_{2}}$, and let $K \subseteq \mathbb{R}^{m_{1}}$ be a regular cone. Consider the primal conic program $\inf \left\{c^{T} x: A_{1} x \succeq_{K} b_{1}, A_{2} x \geq b_{2}\right\}$ and its corresponding dual conic program $\sup \left\{b_{1}^{T} \lambda_{1}+b_{2}^{T} \lambda_{2}: A_{1}^{T} \lambda_{1}+A_{2}^{T} \lambda_{2}=c, \lambda_{1} \succeq_{K_{*}} 0, \lambda_{2} \geq 0\right\}$, where $K_{*}:=\left\{\lambda \in \mathbb{R}^{m}: \lambda^{T} x \geq 0 \forall x \in K\right\}$ is the dual cone to $K$. Then,

(i) weak duality always holds;

(ii) if either (a) the feasible region of the continuous relaxation is bounded, or (b) there exists an essentially strictly feasible point, that is, a point $\hat{x} \in \mathbb{R}^{n}$ such that $A_{1} \hat{x} \succ_{K} b_{1}$ and $A_{2} \hat{x} \geq b_{2}$, then strong duality holds.

2.2. A new sufficient condition for strong duality. Although the strict feasibility conditions in Theorems 2.5 and 2.6 are somewhat analogous, in the MIP case the condition has a crucial limitation: it is not satisfied for conic MIPs that include binary variables and/or have equality constraints in their formulation, or in general if the conic set does not include a mixed-integer point in its interior (see Figure 1). These are very important cases, as conic MIPs with these characteristics often arise when modeling real-life problems.

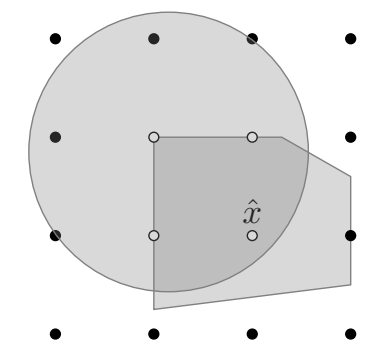

(a) There exists a strictly feasible mixedinteger point.

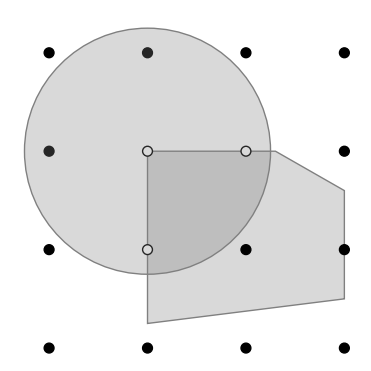

(b) There is no strictly feasible mixed-integer point.

FIG. 1. Two cases comparing the applicability of Theorem 2.5.

In this paper, we generalize all the known sufficient conditions for strong duality by showing that whenever the subadditive dual is feasible, it is a strong dual. Notice that when considering the subadditive dual problem (2), this sufficient condition is the most general possible. Furthermore, we show that this condition is equivalent to feasibility of the conic dual of the continuous relaxation of the conic MIP. We state our result in the theorem below.

Theorem 2.7. Assume that the primal problem (1) is feasible. Then,

(i) if the subadditive dual problem (2) is feasible, then it is a strong dual for (1);

(ii) the subadditive dual (2) is feasible if and only if the conic dual of the continuous relaxation of (1) is feasible.

We obtain the following corollary.

Corollary 2.8. If the primal problem (1) and the conic dual of its continuous relaxation are both feasible, then (2) is a strong dual for (1).

For a feasible conic MIP, notice that the condition in Corollary 2.8 is simpler than the one given in Theorem 2.5, as it only requires checking the feasibility of a conic set rather than finding a mixed-integer point in the interior of a conic set, which is an NP-complete problem even for polyhedral sets [28, Chapter 18.1]. Furthermore, in 
this paper we show that some "natural" conditions for (2) to be a strong dual for (1), including the sufficient conditions known in the literature, actually imply that the conic dual of the continuous relaxation of (1) is feasible (see section 2.4).

2.3. Finiteness property for Dirichlet convex sets. Next we study the finiteness property, a relationship between the finiteness of the optimal value of a general convex MIP and the finiteness of the optimal value of its continuous relaxation.

Definition 2.9 (finiteness property). A convex set $X \subseteq \mathbb{R}^{n_{1}+n_{2}}$ is said to have the finiteness property with respect to $\mathbb{Z}^{n_{1}} \times \mathbb{R}^{n_{2}}$ if for every vector $c \in \mathbb{R}^{n_{1}+n_{2}}$, we have $\sup \left\{c^{T} x: x \in X\right\}<+\infty$ if and only if $\sup \left\{c^{T} x: x \in X \cap\left(\mathbb{Z}^{n_{1}} \times \mathbb{R}^{n_{2}}\right)\right\}<+\infty$.

The finiteness property is closely related to strong duality. A sufficient condition for general convex sets to have this property is stated in [20]. In this paper, we show a more general sufficient condition for the finiteness property to hold and then use it to show the results that we describe in section 2.4. Before stating the new sufficient condition, we need some definitions.

Definition 2.10 (mixed lattices). A mixed lattice is a set of the form

$$
\mathbb{M}=\left\{A x+B y: x \in \mathbb{Z}^{n_{1}}, y \in \mathbb{R}^{n_{2}}\right\}
$$

where $A \in \mathbb{R}^{m \times n_{1}}, B \in \mathbb{R}^{m \times n_{2}}$, and the set $\mathcal{L}=\left\{A x: x \in \mathbb{Z}^{n_{1}}\right\}$ is a lattice contained in $V^{\perp}$, where $V=\left\{B y: y \in \mathbb{R}^{n_{1}}\right\}$. If $\mathbb{M}=\left\{A x: x \in \mathbb{Z}^{n_{1}}\right\}$, that is, there are no "continuous" components, we simply call $\mathbb{M}$ a lattice.

The following definition is a slight modification of the definition of "convex sets with the Dirichlet property" that appears in [19].

Definition 2.11 (Dirichlet convex sets). A convex set $P \subseteq \mathbb{R}^{n}$ is said to be a Dirichlet convex set with respect to a mixed lattice $\mathbb{M}$ if for all $z \in P \cap \mathbb{M}, r \in$ rec.cone $(P)$ and for all $\epsilon>0, \gamma \geq 0$ there exists a point $w \in P \cap \mathbb{M}$ at a (Euclidean) distance less than $\epsilon$ from the half-line $\{z+\lambda r: \lambda \geq \gamma\}$.

Examples of Dirichlet convex sets with respect to $\mathbb{M}=\mathbb{Z}^{n_{1}} \times \mathbb{R}^{n_{2}}$ are, for instance, bounded sets, rational polyhedra, and strictly convex sets (see Proposition 4.5 and Corollary 4.6).

The finiteness property was first proven for rational polyhedra in [18]. A related result in [20] states that if a convex set $X$ contains a mixed-integer point in its interior, then $X$ has the finiteness property (see Proposition 4.5 in [20]). We extend the latter result as follows.

Theorem 2.12. Let $\mathbb{M} \subseteq \mathbb{R}^{n}$ be a mixed lattice, let $c \in \mathbb{R}^{n}$, and let $P \subseteq \mathbb{R}^{n}$ be a Dirichlet convex set with respect to $\mathbb{M}$. Let $S=P \cap \mathbb{M}$ and let $X \subseteq \mathbb{R}^{n}$ be a closed convex set such that $\operatorname{int}(X) \cap S \neq \emptyset$. Then,

$$
\sup _{x \in X \cap S} c^{T} x<+\infty \quad \Leftrightarrow \quad \sup _{x \in X \cap P} c^{T} x<+\infty .
$$

Theorem 2.12 extends the result in [20] from the case in which $\mathbb{M}=\mathbb{Z}^{n_{1}} \times \mathbb{R}^{n_{1}}$ and $P=\mathbb{R}^{n}$ to arbitrary mixed-integer lattices $\mathbb{M}$ and Dirichlet convex sets $P$. 
2.4. Some natural sufficient conditions that imply dual feasibility. Consider the following conic MIP:

$$
\begin{aligned}
z^{*}:=\inf & c^{T} x+d^{T} y \\
\text { s.t. } & A_{1} x+G_{1} y \succeq_{K_{1}} b_{1}, \\
& A_{2} x+G_{2} y \succeq_{K_{2}} b_{2}, \\
& x \in \mathbb{Z}^{n_{1}}, y \in \mathbb{R}^{n_{2}},
\end{aligned}
$$

where $c \in \mathbb{R}^{n_{1}}, d \in \mathbb{R}^{n_{2}}$, and for $i=1,2, K_{i} \subseteq \mathbb{R}^{m_{i}}$ is a regular cone, $A_{i} \in \mathbb{R}^{m_{i} \times n_{1}}$, $G_{i} \in \mathbb{R}^{m_{i} \times n_{2}}, b_{i} \in \mathbb{R}^{m_{i}}$.

Let us define $S_{i}=\left\{(x, y) \in \mathbb{R}^{n_{1}} \times \mathbb{R}^{n_{2}}: A_{i} x+G_{i} y \succeq_{K_{i}} b_{i}\right\}$ for $i=1,2$. As a consequence of Theorems 2.6 and 2.12, we obtain the following result.

THEOREM 2.13. Suppose that the optimal value of (3) is finite, $\operatorname{int}\left(S_{1}\right) \cap S_{2} \cap$ $\left(\mathbb{Z}^{n_{1}} \times \mathbb{R}^{n_{2}}\right) \neq \emptyset$, and one of the following conditions is satisfied:

A. The set $S_{2}$ is bounded.

B. The set $S_{2}$ is a rational polyhedron.

Then, the conic dual of the continuous relaxation of (3) is feasible.

Note that by Corollary 2.8, the assumptions of Theorem 2.13 also imply that the subadditive dual of (3) is a strong dual.

The assumptions in Theorem 2.13 include the following special cases of "natural" sufficient conditions for strong duality of the subadditive dual:

(i) The conic MIP has a bounded feasible region.

(ii) The conic MIP is a linear MIP defined by rational data.

(iii) The conic MIP satisfies the mixed-integer strict feasibility condition in Theorem $2.5[20]$.

(iv) The conic MIP set is mixed-integer essentially strictly feasible, that is, there exists a mixed-integer feasible point that satisfies the nonlinear conic constraints strictly.

(v) The conic MIP includes binary variables and either satisfies (i) or (iv).

Indeed, when A holds we obtain condition (i) and when B holds we obtain condition (iv). Conditions (ii) and (iii) can be seen as the special cases of condition (iv) that we obtain when $S_{1}=\mathbb{R}^{n_{1}} \times \mathbb{R}^{n_{2}}$ and $S_{2}=\mathbb{R}^{n_{1}} \times \mathbb{R}^{n_{2}}$, respectively. Finally, condition (v) is a special case of condition (i) or condition (iv).

We note here that conditions (ii) and (iii) are already known in the literature and that, to the best of our knowledge, conditions (i), (iv), and (v) are new.

3. Proof of Theorem 2.7 and some examples. In this section, we first show an extension of Theorem 2.5 (see Proposition 3.1) and use this result to give a proof for Theorem 2.7. Then, we illustrate the use of our results by giving two examples.

3.1. Strong duality for conic MIPs with binary variables. In this section, we consider the following conic MIP:

$$
\begin{aligned}
z^{*}=\inf & c^{T} x+d^{T} y+h^{T} w \\
\text { s.t. } & A x+G y+H w \succeq_{K} b, \\
& x \in \mathbb{Z}^{n_{1}}, y \in \mathbb{R}^{n_{2}}, w \in\{0,1\}^{n_{3}},
\end{aligned}
$$

where $K \subseteq \mathbb{R}^{m}$ is a regular cone, $c \in \mathbb{R}^{n_{1}}, d \in \mathbb{R}^{n_{2}}, h \in \mathbb{R}^{n_{3}}, A \in \mathbb{R}^{m \times n_{1}}, G \in \mathbb{R}^{m \times n_{2}}$, $H \in \mathbb{R}^{m \times n_{3}}$, and $b \in \mathbb{R}^{m}$.

We show that the subadditive dual of (4) is a strong dual when we require $z^{*}>$ $-\infty$ and strict feasibility of the conic constraints. 
Proposition 3.1. If $z^{*}>-\infty$ and there exists $(\hat{x}, \hat{y}, \hat{w}) \in \mathbb{Z}^{n_{1}} \times \mathbb{R}^{n_{2}} \times\{0,1\}^{n_{3}}$ such that $A \hat{x}+G \hat{y}+H \hat{w} \succ_{K} b$, then the subadditive dual of (4), that is,

$$
\begin{aligned}
& \rho^{*}=\sup F(b, 0,-e) \\
& \text { s.t. } F\left(A^{j}, 0,0\right)=-F\left(-A^{j}, 0,0\right)=c_{j}, \quad j=1, \ldots, n_{1} \text {, } \\
& \bar{F}\left(G^{j}, 0,0\right)=-\bar{F}\left(-G^{j}, 0,0\right)=d_{j}, \quad j=1, \ldots, n_{2}, \\
& F\left(H^{j}, e^{j},-e^{j}\right)=-F\left(-H^{j},-e^{j}, e^{j}\right)=h_{j}, \quad j=1, \ldots, n_{3}, \\
& F(0,0,0)=0 \text {, } \\
& F \in \mathcal{F}_{K \times \mathbb{R}_{+}^{n_{3}} \times \mathbb{R}_{+}^{n_{3}},}
\end{aligned}
$$

is a strong dual. Here, vectors $e$ and $e^{j}$ respectively denote the vector of ones and the jth unit vector in $\mathbb{R}^{n_{3}}$.

Proof. First of all, by construction, (5) is a weak dual for (4) (see [20]). Hence, we have $\rho^{*} \leq z^{*}$.

Let $\epsilon \in(0,1)$ and consider the "perturbed" primal problem

$$
\begin{aligned}
z^{\prime}=\inf & c^{T} x+d^{T} y+h^{T} w \\
\text { s.t. } & A x+G y+H w \succeq_{K} b, \\
& w \geq-\epsilon e, \\
- & w \geq-(1+\epsilon) e, \\
& x \in \mathbb{Z}^{n_{1}}, y \in \mathbb{R}^{n_{2}}, w \in \mathbb{Z}^{n_{3}} .
\end{aligned}
$$

Due to the choice of $\epsilon$, the feasible regions of (4) and (6) coincide. Thus, we obtain that $z^{\prime}=z^{*}$. We also note that point $(\hat{x}, \hat{y}, \hat{w}) \in \mathbb{Z}^{n_{1}} \times \mathbb{R}^{n_{2}} \times\{0,1\}^{n_{3}}$ satisfies all the constraints of (6) strictly. Therefore, we can apply Theorem 2.5 to (6) and its subadditive dual

$$
\begin{array}{rlr}
\rho^{\prime}=\sup & f(b,-\epsilon e,-(1+\epsilon) e) & \\
\text { s.t. } & f\left(A^{j}, 0,0\right)=-f\left(-A^{j}, 0,0\right)=c_{j}, & j=1, \ldots, n_{1}, \\
& \bar{f}\left(G^{j}, 0,0\right)=-\bar{f}\left(-G^{j}, 0,0\right)=d_{j}, & j=1, \ldots, n_{2}, \\
& f\left(H^{j}, e^{j},-e^{j}\right)=-f\left(-H^{j},-e^{j}, e^{j}\right)=h_{j}, & j=1, \ldots, n_{3}, \\
& f(0,0,0)=0, \\
& f \in \mathcal{F}_{K \times \mathbb{R}_{+}^{n_{3}} \times \mathbb{R}_{+}^{n_{3}} .}
\end{array}
$$

Since $z^{\prime}=z^{*}>-\infty$, we conclude that there exists a function $f^{\prime}: \mathbb{R}^{m} \times \mathbb{R}^{n_{3}} \times \mathbb{R}^{n_{3}} \rightarrow \mathbb{R}$ that is an optimal solution to $(7)$ and such that $f^{\prime}(b,-\epsilon e,-(1+\epsilon) e)=\rho^{\prime}=z^{\prime}$. Since the feasible regions of (5) and (7) are the same, the function defined as $f^{\prime}$ is also a feasible solution to (5). Also, we have

$$
\rho^{*} \geq f^{\prime}(b, 0,-e) \geq f^{\prime}(b,-\epsilon e,-(1+\epsilon) e)=\rho^{\prime}=z^{\prime}=z^{*},
$$

where the first inequality follows since $f^{\prime}$ is a feasible solution to (5), and the last inequality follows due to the fact that $f^{\prime}$ is a nondecreasing function with respect to $K \times \mathbb{R}_{+}^{n_{3}} \times \mathbb{R}_{+}^{n_{3}}$. Combining the previous facts, we conclude that $z^{*}=\rho^{*}$ and (5) is solvable, proving that it is a strong dual. 
3.2. Strong duality for conic MIPs with feasible subadditive dual. Before giving the proof of Theorem 2.7 we introduce some notation. For any $u \in \mathbb{R}^{m}$ we define

$$
\vartheta_{M I P}^{*}(u):=\inf \left\{c^{T} x+d^{T} y: A x+G y \succeq_{K} u, x \in \mathbb{Z}^{n_{1}}, y \in \mathbb{R}^{n_{2}}\right\} .
$$

The function $\vartheta_{M I P}^{*}: \mathbb{R}^{m} \rightarrow \mathbb{R} \cup\{-\infty,+\infty\}$ is the value function of (1) and, in particular, $\vartheta_{M I P}^{*}(b)=z^{*}, \vartheta_{M I P}^{*}(u)=-\infty$ when the objective function of the conic MIP is not bounded below and $\vartheta_{M I P}^{*}(u)=+\infty$ when the conic MIP is infeasible.

Proof of Theorem 2.7. (i) First of all, by construction, (2) is a weak dual for (1) (see [20]). Hence, we have $\rho^{*} \leq z^{*}$. Also, observe that $z^{*}>-\infty$, as the dual (2) is feasible and any dual feasible solution provides a lower bound for the optimal value of the primal (1).

Now, let $(\hat{x}, \hat{y}) \in \mathbb{Z}^{n_{1}} \times \mathbb{R}^{n_{2}}$ be a feasible solution to the primal (1) and let $v \in K$ such that $A \hat{x}+G \hat{y}+v \succ_{K} b$ (for instance, any $v \succ_{K} 0$ satisfies this condition). Since $v \in K$, we have that any feasible point for the primal (1) is also feasible for the primal with right-hand side $b-v$ (as $b \succeq_{K} b-v$ ), and thus $\vartheta_{M I P}^{*}(b-v) \leq \vartheta_{M I P}^{*}(b)=z^{*}$. On the other hand, since the subadditive dual (2) is feasible, we obtain that the subadditive dual for the primal problem with right-hand side $b-v$ is also feasible (as feasibility of the subadditive dual does not depend on the right-hand side of the primal), and hence by weak duality we have $\vartheta_{M I P}^{*}(b-v)>-\infty$.

Consider the "perturbed" primal problem

$$
\begin{aligned}
z^{\prime}=\inf & c^{T} x+d^{T} y+\left[\vartheta_{M I P}^{*}(b)-\vartheta_{M I P}^{*}(b-v)\right] w \\
\text { s.t. } & A x+G y+v w \succeq_{K} b, \\
& x \in \mathbb{Z}^{n_{1}}, y \in \mathbb{R}^{n_{2}}, w \in\{0,1\} .
\end{aligned}
$$

We have that $z^{\prime}=\min \left\{\vartheta_{M I P}^{*}(b)+0, \vartheta_{M I P}^{*}(b-v)+\left(\vartheta_{M I P}^{*}(b)-\vartheta_{M I P}^{*}(b-v)\right)\right\}=z^{*}$. We also note that the vector $(\hat{x}, \hat{y}, 1) \in \mathbb{Z}^{n_{1}} \times \mathbb{R}^{n_{2}} \times \mathbb{Z}$ satisfies the conic constraint strictly, and hence we can apply Proposition 3.1 to (8) and its subadditive dual

$$
\begin{aligned}
& \rho^{\prime}=\sup f(b, 0,-1) \\
& \text { s.t. } f\left(A^{j}, 0,0\right)=-f\left(-A^{j}, 0,0\right)=c_{j}, \quad j=1, \ldots, n_{1} \text {, } \\
& \bar{f}\left(G^{j}, 0,0\right)=-\bar{f}\left(-G^{j}, 0,0\right)=d_{j}, \quad j=1, \ldots, n_{2}, \\
& f(v, 0,-1)=-f(-v, 0,1)=\vartheta_{M I P}^{*}(b)-\vartheta_{M I P}^{*}(b-v), \\
& f(0,0,0)=0 \text {, } \\
& f \in \mathcal{F}_{K \times \mathbb{R}_{+} \times \mathbb{R}_{+}} \text {. }
\end{aligned}
$$

Since $z^{\prime}=z^{*}>-\infty$, we conclude that there exists a function $f^{\prime}: \mathbb{R}^{m} \times \mathbb{R} \times \mathbb{R} \rightarrow \mathbb{R}$ that is an optimal solution to (9) and such that $f(b, 0,-1)=\rho^{\prime}=z^{\prime}$. It is easy to show that the function $F: \mathbb{R}^{m} \rightarrow \mathbb{R}$ defined as $F(u):=f^{\prime}(u, 0,0)$ is a feasible solution to (2). Furthermore, we have

$$
\rho^{*} \geq F(b)=f^{\prime}(b, 0,0) \geq f^{\prime}(b, 0,-1)=\rho^{\prime}=z^{\prime}=z^{*},
$$

where the first inequality follows since $F$ is a feasible solution to (2), and the last inequality follows due to the fact that $f^{\prime}$ is a nondecreasing function with respect to $K \times \mathbb{R}_{+} \times \mathbb{R}_{+}$. Combining the previous facts, we conclude that $z^{*}=\rho^{*}$ and (2) is solvable, proving that it is a strong dual. 
(ii) For any $u \in \mathbb{R}^{m}$, we define the conic program

$$
C P(u): \inf \left\{c^{T} x+d^{T} y: A x+G y \succeq_{K} u, x \in \mathbb{R}^{n_{1}}, y \in \mathbb{R}^{n_{2}}\right\},
$$

and denote its value function by $\vartheta_{C P}^{*}(u)$. Note that $C P(b)$ corresponds to the continuous relaxation of (1).

We start by showing that feasibility of the conic dual of $C P(b)$ implies that the subadditive dual is feasible. Indeed, for any $\lambda$ feasible for the conic dual of $C P(b)$, it is easy to check that the function $f: \mathbb{R}^{m} \rightarrow \mathbb{R}$ defined by $f(d)=\lambda^{T} d$ is feasible for (2).

Now we show that feasibility of the subadditive dual (2) implies that the conic dual of $C P(b)$ is feasible. Let $v \succ_{K} 0$. Since the subadditive dual (2) is feasible (independent of the right-hand side of the primal (1)), we have that $\vartheta_{M I P}^{*}(b-v)>-\infty$. Note that $C P(b-v)$ is strictly feasible since the conic MIP (1) is feasible. Therefore, by the finiteness property (Proposition 4.5 in [20]), we have $\vartheta_{C P}^{*}(b-v)>-\infty$ since $\vartheta_{M I P}^{*}(b-v)>-\infty$. Hence, we conclude that $C P(b-v)$ is both strictly feasible and bounded below, implying that its conic dual is feasible due to Theorem 2.6. Finally, since the feasible regions of the conic dual of $C P(b-v)$ and $C P(b)$ coincide, we conclude that the conic dual of $C P(b)$ is feasible.

We note here that although the assumptions in Proposition 3.1 can be shown to be a special case of the assumption in Theorem 2.7 (see section 2.4), we decided to prove Proposition 3.1 separately since on one hand it simplifies the proof of Theorem 2.7, and on the other hand the proof technique is slightly different: in the proof of Proposition 3.1 we perturb the conic MIP (4) only by changing the right-hand side vector whereas in the proof of Theorem 2.7 we perturb the primal (1) by introducing an auxiliary variable with an appropriate objective function coefficient. This difference is also noticeable on how we retrieve the optimal dual function of the conic MIP (4) (resp., (2)) from the optimal solution of the subadditive dual of the perturbed conic MIP (7) (resp., (9)): the optimal dual solution to (4) is exactly the same optimal solution to (7), and the optimal dual solution to (2) is a restriction of the solution to $(9)$.

3.3. Examples. The following examples, which are adapted from [6], feature two feasible, below bounded conic MIPs. In Example 3.2, the subadditive dual is infeasible (hence, the duality gap is infinite). In Example 3.3, the conic MIP is not strictly mixed-integer feasible (therefore, Theorem 2.5 is not applicable) but its subadditive dual is a strong dual since it is feasible.

Example 3.2. Let $L^{3}:=\left\{x \in \mathbb{R}^{3}: \sqrt{x_{1}^{2}+x_{2}^{2}} \leq x_{3}\right\}$ denote the three-dimensional Lorentz cone. Consider the following conic MIP:

$$
\begin{array}{ll}
\inf _{x \in \mathbb{Z}^{2}} & x_{2} \\
\text { s.t. } & {\left[\begin{array}{ll}
1 & 0 \\
0 & 1 \\
1 & 0
\end{array}\right]\left[\begin{array}{l}
x_{1} \\
x_{2}
\end{array}\right] \succeq_{L^{3}}\left[\begin{array}{l}
0 \\
0 \\
0
\end{array}\right] .}
\end{array}
$$

Observe that the primal problem (10) is below bounded (since we have $x_{2}=0$ in any feasible solution) but not strictly feasible. We claim that its subadditive dual is 
infeasible. To prove our claim, we consider the perturbed primal problem

$$
\begin{array}{ll}
\inf _{x \in \mathbb{Z}^{2}} & x_{2} \\
\text { s.t. } & {\left[\begin{array}{ll}
1 & 0 \\
0 & 1 \\
1 & 0
\end{array}\right]\left[\begin{array}{l}
x_{1} \\
x_{2}
\end{array}\right] \succeq_{L^{3}}\left[\begin{array}{c}
0 \\
0 \\
-\epsilon
\end{array}\right]}
\end{array}
$$

for $\epsilon>0$. Notice that the perturbed primal problem (11) is unbounded below as any $\left(x_{1}, x_{2}\right)$ with $x_{2} \in \mathbb{Z}_{-}$and $x_{1}=\left\lceil\left(x_{2}^{2}-\epsilon^{2}\right) / 2 \epsilon\right\rceil$ is feasible. Therefore, the subadditive dual of (11) is infeasible, which implies that the subadditive dual of problem (10) is also infeasible as these two subadditive duals share the same feasible region.

Example 3.3. Let $\mathbb{S}_{+}^{3}:=\left\{X \in \mathbb{R}^{3 \times 3}: u^{T} X u \geq 0 \forall u \in \mathbb{R}^{3}\right\}$ denote the cone of $3 \times 3$ positive semidefinite matrices. Consider the conic MIP

$$
\begin{array}{ll}
\inf _{x \in \mathbb{Z}^{2}} & x_{2} \\
\text { s.t. } & {\left[\begin{array}{lll}
0 & 0 & 0 \\
0 & 1 & 0 \\
0 & 0 & 0
\end{array}\right] x_{1}+\left[\begin{array}{lll}
1 & 0 & 0 \\
0 & 0 & 1 \\
0 & 1 & 0
\end{array}\right] x_{2} \succeq_{\mathbb{S}_{+}^{3}}\left[\begin{array}{ccc}
-1 & 0 & 0 \\
0 & 0 & 0 \\
0 & 0 & 0
\end{array}\right],}
\end{array}
$$

and its subadditive dual

$$
\begin{aligned}
& \sup f\left(\left[\begin{array}{ccc}
-1 & 0 & 0 \\
0 & 0 & 0 \\
0 & 0 & 0
\end{array}\right]\right) \\
& \text { s.t. } f\left(\left[\begin{array}{lll}
0 & 0 & 0 \\
0 & 1 & 0 \\
0 & 0 & 0
\end{array}\right]\right)=-f\left(-\left[\begin{array}{lll}
0 & 0 & 0 \\
0 & 1 & 0 \\
0 & 0 & 0
\end{array}\right]\right)=0, \\
& f\left(\left[\begin{array}{lll}
1 & 0 & 0 \\
0 & 0 & 1 \\
0 & 1 & 0
\end{array}\right]\right)=-f\left(-\left[\begin{array}{lll}
1 & 0 & 0 \\
0 & 0 & 1 \\
0 & 1 & 0
\end{array}\right]\right)=1, \\
& f\left(\left[\begin{array}{lll}
0 & 0 & 0 \\
0 & 0 & 0 \\
0 & 0 & 0
\end{array}\right]\right)=0, \\
& f \in \mathcal{F}_{\mathbb{S}_{+}^{3} .}
\end{aligned}
$$

Notice that the primal problem (12) is below bounded (since we have $x_{2}=0$ in any feasible solution) but not strictly feasible. We observe that $\Lambda=e^{1}\left(e^{1}\right)^{T}$ is a feasible solution for the conic dual of the continuous relaxation of $(12)$, where $e^{1}=\left[\begin{array}{lll}1 & 0 & 0\end{array}\right]^{T}$. Therefore, the function $f: \mathbb{R}^{3 \times 3} \rightarrow \mathbb{R}$ defined as $f(A)=\operatorname{Tr}(\Lambda A)=A_{11}$ is a feasible solution for the subadditive dual (13), implying that it is a strong dual.

4. Properties of Dirichlet convex sets and the proof of Theorem 2.12. In this section we first study some properties of Dirichlet convex sets that allow us to show that some important classes of convex sets, such as bounded convex sets, strictly convex sets, and rational polyhedra, are Dirichlet convex sets, and then give a proof of Theorem 2.12 .

4.1. Dirichlet convex sets. We start by stating a lemma on the intersection of mixed lattices and linear subspaces. 
Lemma 4.1 (Lemma 3.13 in [23]). Let $\mathbb{M} \subseteq \mathbb{R}^{n}$ be a mixed lattice and let $W \subseteq \mathbb{R}^{n}$ be a linear subspace. Then $\mathbb{M} \cap W$ is a mixed lattice.

In order to establish a sufficient condition for a convex set to be a Dirichlet convex set, we need the following lemma, which is a consequence of the Dirichlet's Diophantine approximation theorem and appears in this form for the $\mathbb{M}=\mathbb{Z}^{n}$ case in $[5]$.

Lemma 4.2 (Basu et al. [5]). Let $\mathbb{M} \subseteq \mathbb{R}^{n}$ be a mixed lattice. If $z \in \mathbb{M}$ and $r \in \operatorname{aff}(\mathbb{M})$, then for all $\epsilon>0$ and $\gamma \geq 0$, there exists a point of $w \in \mathbb{M}$ at a distance less than $\epsilon$ from the half-line $\{z+\lambda r: \lambda \geq \gamma\}$.

Although the extension to general mixed lattices is a straightforward consequence of the result in [5], for completeness we still present the proof of Lemma 4.2 in Appendix A.

The following result is a special case of a classic result in convex analysis. We present a proof in Appendix A that is adapted from the proof of Theorem 18.1 in [24].

Lemma 4.3. Let $\mathbb{M} \subseteq \mathbb{R}^{n}$ be a mixed lattice and let $X \subseteq \mathbb{R}^{n}$ be a convex set. Then for any face $F$ of $X$, we have $\operatorname{conv}(F \cap \mathbb{M})=\operatorname{conv}(X \cap \mathbb{M}) \cap F$.

The next lemma establishes that the property of being a Dirichlet convex set is invariant under invertible affine mappings that preserve the corresponding mixed lattice.

Lemma 4.4. Let $\mathbb{M} \subseteq \mathbb{R}^{n}$ be a mixed lattice and let $X \subseteq \mathbb{R}^{n}$ be a Dirichlet convex set. Let $T: \mathbb{R}^{n} \rightarrow \mathbb{R}^{n}$ be an invertible affine mapping such that $T(\mathbb{M})=\mathbb{M}$. Then $T(X)$ is a Dirichlet convex set with respect to $\mathbb{M}$.

Proof. Let $T(x)=A x+b$, where $A$ is an invertible $n \times n$ matrix and $b \in \mathbb{R}^{n}$.

First, observe that since $X$ is a convex set and $T$ is an affine mapping, $T(X)=$ $A X+b$ is indeed a convex set.

We now show that $T(X)$ is a Dirichlet convex set. Let $y \in T(X) \cap \mathbb{M}, s \in$ rec.cone $(T(X)), \epsilon>0$, and $\gamma \geq 0$. We must show that there exists a point $v \in$ $T(X) \cap \mathbb{M}$ at a distance less than $\epsilon$ from the half-line $\{y+\lambda s: \lambda \geq \gamma\}$.

Since $T$ is invertible and $T(\mathbb{M})=\mathbb{M}$, there exists $z \in X \cap \mathbb{M}$ such that $T(z)=y$. Moreover, due to the fact that $T$ is an affine mapping, there exists $r \in \operatorname{rec} . \operatorname{cone}(X)$ such that $A r=s$.

Since $X$ is a Dirichlet convex set with respect to $\mathbb{M}$, we have that there exists a point $w \in X \cap \mathbb{M}$ at a distance less than $\epsilon /\|A\|_{2}$ from the half-line $\{z+\lambda r: \lambda \geq \gamma\}$, that is, $\left\|w-z-\lambda^{*} r\right\|_{2} \leq \epsilon /\|A\|_{2}$ for some $\lambda^{*} \geq \gamma$. Let $v=T(w)$ and notice that $v \in T(X) \cap \mathbb{M}$ as $w \in X \cap \mathbb{M}$. We obtain that

$$
\begin{aligned}
\left\|v-y-\lambda^{*} s\right\|_{2} & =\left\|T(w)-T(z)-\lambda^{*} A r\right\|_{2} \\
& =\left\|A w+b-A z-b-\lambda^{*} A r\right\|_{2} \\
& \leq\|A\|_{2}\left\|w-z-\lambda^{*} r\right\|_{2} \\
& \leq \epsilon .
\end{aligned}
$$

This implies that the distance between $v$ and the half-line $\{y+\lambda s: \lambda \geq \gamma\}$ is less than $\epsilon$. Therefore, we conclude that $T(X)$ is a Dirichlet convex set with respect to $\mathbb{M}$.

We remark that not all operations preserve the property of being a Dirichlet convex set. For instance, the intersection of two Dirichlet convex sets is not necessarily a Dirichlet convex set; see Example 2.9 in [23] for an illustration of this fact. 
The most important result in this section is a sufficient condition for a convex set to be a Dirichlet convex set, which we state in the next proposition.

Proposition 4.5. Let $\mathbb{M} \subseteq \mathbb{R}^{n}$ be a mixed lattice and let $X \subseteq \mathbb{R}^{n}$ be a convex set such that rec.cone $(X)=$ rec.cone $(\operatorname{conv}(X \cap \mathbb{M}))$. Then $X$ is a Dirichlet convex set with respect to $\mathbb{M}$.

Proof. Let $z \in X \cap \mathbb{M}$. Then by Lemma 4.4 applied to $\mathbb{M}, X$, and $T(x)=x-z$, we conclude that the set $X$ is a Dirichlet convex set with respect to $\mathbb{M}$ if and only if $X-z$ is a Dirichlet convex set with respect to $\mathbb{M}$. Therefore, we may assume for the rest of the proof that the set $X$ contains the origin, and thus $W=\operatorname{aff}(X \cap \mathbb{M})$ is a linear subspace (we will use this latter fact in order to apply Lemma 4.1 in Case 1 below).

Let $z \in X \cap \mathbb{M}, r \in$ rec.cone $(X), \epsilon>0$, and $\gamma \geq 0$. We must show that there exists a point $w \in X \cap \mathbb{M}$ at a distance less than $\epsilon$ from the half-line $\{z+\lambda r: \lambda \geq \gamma\}$.

We will use induction on the dimension of $X$. Clearly, if $\operatorname{dim}(X)=0$, the result is true. Now, we assume that any convex set $X^{\prime} \subseteq \mathbb{R}^{n}$ with $\operatorname{dim}\left(X^{\prime}\right)<\operatorname{dim}(X)$ such that rec.cone $\left(X^{\prime}\right)=\operatorname{rec} \cdot \operatorname{cone}\left(\operatorname{conv}\left(X^{\prime} \cap \mathbb{M}\right)\right)$ is a Dirichlet convex set with respect to M. We have two cases.

Case 1 (the half-line $\left\{z+\lambda r: \lambda \geq \gamma^{\prime}\right\}$ is contained in the relative interior of $X$ for some $\left.\gamma^{\prime} \geq \gamma\right)$. Since $\left\{z+\lambda r: \lambda \geq \gamma^{\prime}\right\}$ is contained in the relative interior of $X$, there exists $\epsilon^{\prime}>0$ such that $\epsilon^{\prime}<\epsilon$ and any point in $\operatorname{aff}(X)$ at distance less than $\epsilon^{\prime}$ from the half-line $\left\{z+\lambda r: \lambda \geq \gamma^{\prime}\right\}$ belongs to $X$.

Recall the linear subspace $W=\operatorname{aff}(X \cap \mathbb{M})$ and let $\mathbb{M}^{\prime}=\mathbb{M} \cap W$. By Lemma 4.1, we obtain that $\mathbb{M}^{\prime} \subseteq W$ is a mixed lattice. Moreover, by definition of $W$ and $\mathbb{M}^{\prime}$, we obtain $W=\operatorname{aff}\left(\mathbb{M}^{\prime}\right)$, and since $r \in \operatorname{rec} \cdot \operatorname{cone}(X)=\operatorname{rec} \cdot \operatorname{cone}(\operatorname{conv}(X \cap \mathbb{M}))$ and $X \cap \mathbb{M} \subseteq W$ we obtain that $r \in \operatorname{rec} \cdot \operatorname{cone}(W)=\operatorname{aff}\left(\mathbb{M}^{\prime}\right)$. On the other hand, since $z \in X \cap \mathbb{M}$, we obtain $z \in \mathbb{M}^{\prime}$. We can apply Lemma 4.2 and conclude that there exists a point $w \in \mathbb{M}^{\prime}$ at a distance less than $\epsilon^{\prime}$ from the half-line $\left\{z+\lambda r: \lambda \geq \gamma^{\prime}\right\}$. Since $w \in \mathbb{M}^{\prime}$, we obtain that $w \in W \subseteq \operatorname{aff}(X)$, and therefore, by the selection of $\epsilon^{\prime}$ and since $\gamma^{\prime} \geq \gamma$, we conclude that $w \in X \cap \mathbb{M}$ and that $w$ is at distance less than $\epsilon$ from the half-line $\{z+\lambda r: \lambda \geq \gamma\}$.

Case 2 (the half-line $\{z+\lambda r: \lambda \geq \gamma\}$ is contained in a proper face $F$ of $X$ ). Since $F$ is a proper face of $X$, we have $\operatorname{dim}(F)<\operatorname{dim}(X)$. Moreover, by Lemma 4.3, we obtain that $\operatorname{conv}(F \cap \mathbb{M})=\operatorname{conv}(X \cap \mathbb{M}) \cap F$. Furthermore, since $\operatorname{conv}(F \cap \mathbb{M}) \neq \emptyset$, we obtain

$$
\begin{aligned}
\operatorname{rec} . \operatorname{cone}(\operatorname{conv}(F \cap \mathbb{M})) & =\operatorname{rec} \cdot \operatorname{cone}(\operatorname{conv}(X \cap \mathbb{M})) \cap \operatorname{rec} . \operatorname{cone}(F) \\
& =\operatorname{rec} \cdot \operatorname{cone}(X) \cap \operatorname{rec} \cdot \operatorname{cone}(F) \\
& =\operatorname{rec} \cdot \operatorname{cone}(F) .
\end{aligned}
$$

Therefore, we can apply the induction hypothesis to $F$ and conclude that $F$ is a Dirichlet convex set with respect to $\mathbb{M}$. Since the half-line $\{z+\lambda r: \lambda \geq \gamma\}$ is contained in $F$, we obtain that $z \in F \cap \mathbb{M}$ and that $r \in \operatorname{rec} . \operatorname{cone}(F)$. Therefore, we conclude that there exists a point $w \in F \cap \mathbb{M} \subseteq X \cap \mathbb{M}$ at a distance less than $\epsilon$ from the half-line $\{z+\lambda r: \lambda \geq \gamma\}$.

When the mixed-integer lattice is $\mathbb{M}=\mathbb{Z}^{n_{1}} \times \mathbb{R}^{n_{2}}$, some examples of convex sets $X$ satisfying the assumption rec.cone $(X)=\operatorname{rec} . \operatorname{cone}(\operatorname{conv}(X \cap \mathbb{M}))$ in Proposition 4.5 are bounded convex sets, rational polyhedra [18], closed strictly convex sets [13, 23], and closed convex sets whose recession cone is generated by vectors in $\mathbb{M}$ (see Corollary 1 
in [13] for a proof of this assertion in the $\mathbb{M}=\mathbb{Z}^{n}$ case and the recession cone of the convex set being a rational polyhedral cone). Based on the discussion above, we obtain the following corollary of Proposition 4.5.

Corollary 4.6. The following are Dirichlet convex sets with respect to $\mathbb{Z}^{n_{1}} \times$ $\mathbb{R}^{n_{2}}$ : bounded convex sets, rational polyhedra, closed strictly convex sets, and closed convex sets whose recession cone is generated by vectors in $\mathbb{Z}^{n_{1}} \times \mathbb{R}^{n_{2}}$.

If a convex set $X$ does not contain lines, then Proposition 4.5 can be seen as an extension of Proposition 4.7 in [19]. Indeed, if rec.cone $(X)=\operatorname{rec} \cdot \operatorname{cone}(\operatorname{conv}(X \cap$ $\mathbb{M})$ ) holds, then $\operatorname{conv}(X \cap \mathbb{M})$ is closed (see Theorem 3.20 in [23]). Conversely, if a closed convex set $X$ satisfies that $\operatorname{conv}(X \cap \mathbb{M})$ is a closed set and contains a mixed-lattice vector in its relative interior, then it can be shown that rec.cone $(X)=$ rec.cone $(\operatorname{conv}(X \cap \mathbb{M}))$ (see Theorem 2 in [13] for the proof of this result when $\mathbb{M}=\mathbb{Z}^{n}$ and the convex set is full-dimensional).

4.2. The finiteness property. Let $S \subseteq \mathbb{R}^{n}$. A full-dimensional convex set $Q \subseteq \mathbb{R}^{n}$ is said to be an $S$-free convex set if $\operatorname{int}(Q) \cap S=\emptyset$. $Q$ is said to be a maximal $S$-free convex set if it is not strictly contained in any other $S$-free convex set. When $S=P \cap \mathbb{M}$, where $P$ is a convex set and $\mathbb{M}$ is a mixed lattice, Averkov [2] showed that maximal $S$-free convex sets are polyhedra (see Theorem 2.4 in [2]).

The following lemma gives a property of maximal $S$-free sets in the case in which $S$ is defined as the mixed-lattice points contained in a Dirichlet convex set. This lemma is crucial in the proof of Theorem 2.12.

Lemma 4.7. Let $\mathbb{M} \subseteq \mathbb{R}^{n}$ be a mixed lattice and $P \subseteq \mathbb{R}^{n}$ be a Dirichlet convex set with respect to $\mathbb{M}$. Let $S=P \cap \mathbb{M}$, and let $Q$ be a full-dimensional maximal $S$-free convex set. If $r \in \operatorname{rec} . \operatorname{cone}(P \cap Q)$, then $-r \in \operatorname{rec} . \operatorname{cone}(Q)$.

Proof. Let $Q^{\prime}=\{x-\lambda r: x \in Q, \lambda \geq 0\}$. In order to prove the lemma, we will show that $Q^{\prime}=Q$. Since $Q^{\prime}$ is a convex set, $Q \subseteq Q^{\prime}$, and $Q$ is a maximal $S$-free convex set, it suffices to show that $Q^{\prime}$ is an $S$-free convex set. Assume for a contradiction that $\operatorname{int}\left(Q^{\prime}\right) \cap S \neq \emptyset$. Then there exists $x \in \operatorname{int}(Q)$ and $\gamma \geq 0$ such that $z=x-\gamma r \in S$. Since $x \in \operatorname{int}(Q)$ and $r \in \operatorname{rec}$.cone $(Q)$, there exists $\epsilon>0$ such that the set $\mathcal{H}_{\varepsilon}:=\{x+\lambda r: \lambda \geq 0\}+B(0, \epsilon)$ is contained in $\operatorname{int}(Q)$. On the other hand, since $z \in S$ and $P$ is a Dirichlet convex set with respect to $\mathbb{M}$, we have that there exists a point $z^{\prime} \in S$ at a distance less than $\epsilon$ from the half-line $\{z+\lambda r: \lambda \geq \gamma\}$. Since $\{z+\lambda r: \lambda \geq \gamma\}=\{x+\lambda r: \lambda \geq 0\}$, we obtain that $z^{\prime} \in \mathcal{H}_{\varepsilon} \subseteq \operatorname{int}(Q)$, a contradiction with the fact that $Q$ is an $S$-free set. Therefore, we conclude that $Q^{\prime}=Q$, as desired.

Now we are ready to prove Theorem 2.12.

Proof of Theorem 2.12. We only need to show that $\sup \left\{c^{T} x: x \in X \cap S\right\}<+\infty$ implies $\sup \left\{c^{T} x: x \in X \cap P\right\}<+\infty$.

Let $z^{*}=\sup \left\{c^{T} x: x \in X \cap S\right\}$ and let $X^{\geq}=\left\{x \in X: c^{T} x \geq z^{*}\right\}$. Assume for a contradiction that $\sup \left\{c^{T} x: x \in X \cap P\right\}=+\infty$. Then $X^{\geq}$is a full-dimensional unbounded $S$-free convex set. Therefore, there exists a full-dimensional maximal $S$ free polyhedron $Q \supseteq X^{\geq}$(by Theorem 2.4 in [2]).

On the other hand, since $X$ is not $S$-free, we obtain $X \nsubseteq Q$, so there exists $x_{0} \in X \backslash Q$. In particular, as $Q$ is a polyhedron, there exists an inequality $a^{T} x \leq b$ of $Q$ such that $a^{T} x_{0}>b$. Also notice that since $x_{0} \notin Q$, we have $x_{0} \notin X^{\geq}$and thus $c^{T} x_{0}<z^{*}$. 
Let $\left\{x_{k}\right\}_{k \geq 1} \subseteq X^{\geq} \cap P$ be such that $c^{T} x \rightarrow+\infty$ and for each $k \geq 1$ define

$$
y_{k}=\left[\frac{c^{T} x_{k}-z^{*}}{c^{T} x_{k}-c^{T} x_{0}}\right] x_{0}+\left[\frac{z^{*}-c^{T} x_{0}}{c^{T} x_{k}-c^{T} x_{0}}\right] x_{k} ;
$$

this sequence is well defined since we have $c^{T} x_{k} \geq z^{*}>c^{T} x_{0}$ for all $k \geq 1$.

Notice that for all $k \geq 1$,

$$
c^{T} y_{k}=\frac{c^{T} x_{0}\left(c^{T} x_{k}-z^{*}\right)+c^{T} x_{k}\left(z^{*}-c^{T} x_{0}\right)}{c^{T} x_{k}-c^{T} x_{0}}=z^{*},
$$

and that $y_{k} \in X$ as it is a convex combination of $x_{0} \in X$ and $x_{k} \in X \geq \subseteq X$, and $X$ is a convex set.

Now as $X \geq \cap P \subseteq P \cap Q$, we obtain rec.cone $\left(X^{\geq} \cap P\right) \subseteq \operatorname{rec}$.cone $(P \cap Q)$. Let $L=\operatorname{aff}\left(\operatorname{rec} . \operatorname{cone}\left(X^{\geq} \cap P\right)\right)$ and notice that $L$ is a linear subspace. Then, by Lemma 4.7, we obtain

$$
\left(X^{\geq} \cap P\right)+L \subseteq Q .
$$

Moreover, by the definition of the linear subspace $L$, we have that $\left(X^{\geq} \cap P\right) \cap L^{\perp}$ is a bounded set. Therefore, for any $f \in \mathbb{R}^{n}, \sup \left\{f^{T} x: x \in\left(X^{\geq} \cap P\right)+L\right\}=+\infty$ if and only if there exists $l \in L$ such that $f^{T} l>0$ if and only if inf $\left\{f^{T} x: x \in\left(X^{\geq} \cap P\right)+L\right\}=$ $-\infty$. Since $a^{T} x \leq b$ is a valid inequality for $\left(X^{\geq} \cap P\right)\left(\right.$ as $\left.\left(X^{\geq} \cap P\right)+L \subseteq Q\right)$, the latter properties imply that $a^{T} x \geq b-\eta$ is valid for $\left(X^{\geq} \cap P\right)+L$ for some $\eta>0$.

Observe that

$$
\begin{aligned}
a^{T} y_{k} & =\frac{a^{T} x_{0}\left(c^{T} x_{k}-z^{*}\right)+a^{T} x_{k}\left(z^{*}-c^{T} x_{0}\right)}{c^{T} x_{k}-c^{T} x_{0}} \\
& >\frac{b\left(c^{T} x_{k}-z^{*}\right)+(b-\eta)\left(z^{*}-c^{T} x_{0}\right)}{c^{T} x_{k}-c^{T} x_{0}} \\
& \geq b+\frac{-\eta z^{*}+\eta c^{T} x_{0}}{c^{T} x_{k}-c^{T} x_{0}}
\end{aligned}
$$

where the first inequality follows from (i) $a^{T} x_{0}>b$ and $c^{T} x_{k} \geq z^{*}$, and (ii) $a^{T} x_{k} \geq$ $b-\eta$ (since $\left\{x_{k}\right\}_{k \geq 1} \subseteq X^{\geq} \cap P$ and $a^{T} x \geq b-\eta$ is valid for $\left(X^{\geq} \cap P\right)+L$ ) and $z^{*}>c^{T} x_{0}$ (since $x_{0} \notin Q$, and thus $x \notin X^{\geq}$).

Therefore, as $\frac{-\eta z^{*}+\eta c^{T} x_{0}}{c^{T} x_{k}-c^{T} x_{0}} \rightarrow 0$ as $k \rightarrow+\infty$, for large enough $\bar{K} \geq 1$, we have $a^{T} y_{\bar{K}}>b$. On the other hand, since $y_{\bar{K}} \in X$ and $c^{T} y_{\bar{K}}=z^{*}$ we obtain $y_{\bar{K}} \in X \geq$. Thus, since $X^{\geq} \subseteq Q, y_{\bar{K}} \in Q$ and therefore $a^{T} y_{\bar{K}} \leq b$, a contradiction.

5. Proof of Theorem 2.13. The proof of Theorem 2.13 is a consequence of Theorems 2.6 and 2.12 .

Proof of Theorem 2.13. We first recall conditions A and B in Theorem 2.13:

A. The set $S_{2}$ is bounded.

B. The set $S_{2}$ is a rational polyhedron.

We will show that if the optimal value of (3) is finite, $\operatorname{int}\left(S_{1}\right) \cap S_{2} \cap\left(\mathbb{Z}^{n_{1}} \times \mathbb{R}^{n_{2}}\right) \neq \emptyset$, and one of the assumptions above is satisfied, then the conic dual of the continuous relaxation of (3) is feasible. Observe that under either assumption A or assumption B the set $S_{2}$ is a Dirichlet convex set with respect to the mixed lattice $\mathbb{Z}^{n_{1}} \times \mathbb{R}^{n_{2}}$ (see Corollary 4.6). Therefore, we can use Theorem 2.12 with $X=S_{1}, P=S_{2}$, and 
$\mathbb{M}=\mathbb{Z}^{n_{1}} \times \mathbb{R}^{n_{2}}$ to conclude that the optimal value of the continuous relaxation of (3) is finite.

Now, since the nonlinear conic constraints in (3) are strictly feasible (that is, $\left.\operatorname{int}\left(S_{1}\right) \cap S_{2} \neq \emptyset\right)$ and either A or B is satisfied, then we have that either condition (a) or condition (b) in Theorem 2.6 holds. Therefore, the continuous relaxation of (3) and its conic dual satisfy strong duality. Moreover, since the optimal value of the continuous relaxation of (3) is finite, we conclude that the conic dual of the continuous relaxation of (3) is solvable, and therefore it must be feasible.

6. Final remarks. By weak duality, any function $F \in \mathcal{F}_{K}$ (that is, $F$ subadditive and nondecreasing with respect to the cone $K$ ) that satisfies $F(0)=0$ is a cut-generating function (see, for instance, [11]) and, in particular, defines the following valid inequality for the feasible region of the conic MIP (1):

$$
\sum_{j=1}^{n_{1}} F\left(A^{j}\right) x_{j}+\sum_{j=1}^{n_{2}} \bar{F}\left(G^{j}\right) y_{j} \geq F(b) .
$$

Conversely, the strong duality result in Theorem 2.7 yields the following corollary.

Corollary 6.1. Let $\pi^{T} x+\gamma^{T} y \geq \pi_{o}$ be a valid inequality for the feasible region of the conic MIP (1) and suppose that there exists a subadditive function $f \in \mathcal{F}_{K}$ satisfying $f(0)=0, f\left(A^{j}\right)=-f\left(-A^{j}\right)=\pi_{j}, j=1, \ldots, n_{1}$, and $\bar{f}\left(G^{j}\right)=-\bar{f}\left(-G^{j}\right)=$ $\gamma_{j}, j=1, \ldots, n_{2}$. Then, there exists a subadditive function $F \in \mathcal{F}_{K}$ such that $F(0)=$ $0, F\left(A^{j}\right)=-F\left(-A^{j}\right)=\pi_{j}, j=1, \ldots, n_{1}, \bar{F}\left(G^{j}\right)=-\bar{F}\left(-G^{j}\right)=\gamma_{j}, j=1, \ldots, n_{2}$, and $F(b) \geq \pi_{o}$. In particular, the valid inequality of the form (14) defined by $F$ dominates $\pi^{T} x+\gamma^{T} y \geq \pi_{o}$.

A similar result was proven in [20] (see Corollary 6.1). We emphasize here that we could use the value function of the conic MIP (1), $\vartheta_{M I P}^{*}: \mathbb{R}^{m} \rightarrow \mathbb{R} \cup\{-\infty,+\infty\}$, to generate a valid inequality of the form (14) (see Proposition 4.8 in [20]). Furthermore, by appropriately changing the objective function in (1), and considering the associated value function, we could generate all valid inequalities for (1) in this way. However, one disadvantage of this approach as compared to using functions in $\mathcal{F}_{K}$ is that value functions of conic MIPs are difficult to compute. Moreover, as value functions are in general not finite-valued everywhere, they cannot be cut-generating functions (by definition).

As a final remark, we can combine the proofs of Theorem 3.1, Theorem 2.7, and the derivation in [20] (see the proof of Proposition 5.3) to give an optimal solution of the subadditive dual problem of (1) that is, in fact, the value function of a particular conic MIP. We rigorously state this result in the following corollary.

Corollary 6.2. If the conic MIP (1) and its subadditive dual are both feasible, then there exists an optimal dual function $f^{*}: \mathbb{R}^{m} \rightarrow \mathbb{R}$ that is the value function of a particular conic MIP. More precisely,

$$
\begin{aligned}
& f^{*}(u)=\inf c^{T} x+d^{T} y+\left[\vartheta_{M I P}^{*}(b)-\vartheta_{M I P}^{*}(b-v)\right] w+\left[\vartheta_{M I P}^{*}(b)-2 \Theta^{*}\right] s
\end{aligned}
$$

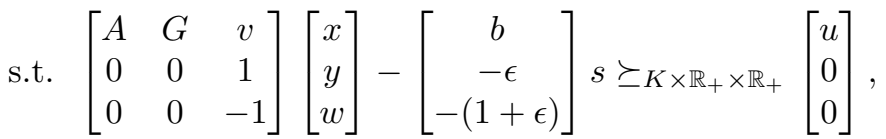

$$
\begin{aligned}
& x \in \mathbb{Z}^{n_{1}}, y \in \mathbb{R}^{n_{2}}, w \in \mathbb{Z}, s \in \mathbb{Z},
\end{aligned}
$$


where $\epsilon \in(0,1), v \in \operatorname{int}(K)$, and

$$
\begin{aligned}
\Theta^{*}=\inf & c^{T} x+d^{T} y+\left[\vartheta_{M I P}^{*}(b)-\vartheta_{M I P}^{*}(b-v)\right] w \\
\text { s.t. } & A x+G y+v w \succeq_{K} b, \\
& w \geq-\epsilon, \\
- & w \geq-(1+\epsilon), \\
& x \in \mathbb{R}^{n_{1}}, y \in \mathbb{R}^{n_{2}}, w \in \mathbb{R} .
\end{aligned}
$$

Appendix A. Omitted proofs. Here, we present the proofs of Lemmas 4.2 and 4.3 from section 4.1 .

Proof of Lemma 4.2. We must show that there exists a point $w \in \mathbb{M}$ at a distance less than $\epsilon$ from the half-line $\{z+\lambda r: \lambda \geq \gamma\}$. We divide the proof into two cases.

Case 1 ( $\mathbb{M}$ is a lattice). In this case, there exists $A$, an $n \times k$ matrix with linearly independent columns such that

$$
\mathbb{M}=\left\{A z: z \in \mathbb{Z}^{k}\right\}
$$

Since $A$ has linearly independent columns, $z \in \mathbb{M}$, and $r \in \operatorname{aff}(\mathbb{M})$, there exist $z^{\prime} \in \mathbb{Z}^{k}$ such that $z=A z^{\prime}$ and $r^{\prime} \in \mathbb{R}^{k}$ such that $r=A r^{\prime}$. By applying the result by Basu et al. [5] for the case $\mathbb{M}=\mathbb{Z}^{k}$, we obtain that there exists a point $w^{\prime} \in \mathbb{M}$ at a distance less than $\epsilon /\|A\|_{2}$ from the half-line $\left\{z^{\prime}+\lambda r^{\prime}: \lambda \geq \gamma\right\}$. In particular, there exists an $l^{\prime}$ in this half-line such that $\left\|w^{\prime}-l^{\prime}\right\|_{2} \leq \epsilon /\|A\|_{2}$. This implies that $\left\|A w^{\prime}-A l^{\prime}\right\|_{2} \leq\|A\|_{2}\left\|w^{\prime}-l^{\prime}\right\|_{2} \leq \epsilon$. As $w:=A w^{\prime} \in \mathbb{M}$ and $A l^{\prime}$ belongs to the half-line $\{z+\lambda r: \lambda \geq \gamma\}$, we conclude the proof.

Case $2(\mathbb{M}$ is a general mixed lattice). Let $\mathcal{L} \subseteq \mathbb{M}$ be any lattice such that $z \in \mathcal{L}$. By Case 1 , there exist $w \in \mathcal{L}$ at a distance less than $\epsilon$ from the half-line $\{z+\lambda r: \lambda \geq \gamma\}$. As $w \in \mathbb{M}$, we are done.

Proof of Lemma 4.3. Clearly, $\operatorname{conv}(F \cap \mathbb{M}) \subseteq \operatorname{conv}(K \cap \mathbb{M}) \cap F$. We will prove the other inclusion. Let $x \in \operatorname{conv}(K \cap \mathbb{M}) \cap F$; we will show that $x \in \operatorname{conv}(F \cap \mathbb{M})$. We have that $x=\lambda_{1} z_{1}+\cdots+\lambda_{p} z_{p}$ for some $z_{1}, \ldots, z_{p} \in K \cap \mathbb{M}$ and $0<\lambda_{1}, \ldots, \lambda_{p} \leq 1$ such that $\lambda_{1}+\cdots+\lambda_{p}=1$. Let $D=\operatorname{conv}\left(\left\{z_{1}, \ldots, z_{p}\right\}\right)$ and observe that $x$ belongs to the relative interior of $D$. It follows that for any $y \in D$, there exists $y^{\prime} \in D$ such that $x=\lambda y+(1-\lambda) y^{\prime}$. Since $y, y^{\prime} \in K, x \in F$, and $F$ is a face of $K$, we obtain that $y, y^{\prime} \in F$. As $y \in D$ was arbitrary, we obtain that $D \subseteq F$ and, in particular, $z_{1}, \ldots, z_{p} \in F$. Therefore, we conclude that $x \in \operatorname{conv}(F \cap \mathbb{M})$, as desired.

Acknowledgment. The authors thank one anonymous referee for some suggestions that improved the presentation and readability of this paper.

\section{REFERENCES}

[1] A. Atamtürk and V. Narayanan, Conic mixed-integer rounding cuts, Math. Program., 122 (2010), pp. 1-20.

[2] G. Averkov, On maximal $S$-free sets and the Helly number for the family of S-convex sets, SIAM J. Discrete Math., 27 (2013), pp. 1610-1624, https://doi.org/10.1137/110850463.

[3] M. Baes, T. Oertel, and R. Weismantel, Duality for mixed-integer convex minimization, Math. Program., 158 (2016), pp. 547-564.

[4] A. Basu, M. Conforti, G. CornuÉjols, R. Weismantel, and S. Weltge, Optimality certificates for convex minimization and Helly numbers, Oper. Res. Lett., 45 (2017), pp. 671-674.

[5] A. Basu, M. Conforti, G. Cornuéjols, and G. Zambelli, Maximal lattice-free convex sets in linear subspaces, Math. Oper. Res., 35 (2010), pp. 704-720. 
[6] A. Ben-Tal and A. Nemirovski, Lectures on Modern Convex Optimization, MOS-SIAM Ser. Optim. 2, SIAM, Philadelphia, PA, 2001.

[7] R. Bixby and E. Rothberg, Progress in computational mixed integer programming - A look back from the other side of the tipping point, Ann. Oper. Res., 149 (2007), pp. 37-41.

[8] R. Brandenberg And L. Roth, New algorithms for $k$-center and extensions, in Combinatorial Optimization and Applications, Lecture Notes in Comput. Sci. 5165, B. Yang, D. Du, and C. Wang, eds., Springer, Berlin, 2008, pp. 64-78, https://doi.org/10.1007/ 978-3-540-85097-7_7.

[9] M. T. ÇezIK and G. Iyengar, Cuts for mixed 0-1 conic programming, Math. Program., 104 (2005), pp. 179-202.

[10] Y. Chen And M. Chen, Extended duality for nonlinear programming, Comput. Optim. Appl., 47 (2010), pp. 33-59.

[11] M. Conforti, G. Cornú́jols, A. Danillidis, C. Lemaréchal, and J. Malick, CutGenerating Functions and S-Free Sets, Math. Oper. Res., 40 (2015), pp. 276-391.

[12] R. Dai And M. Mesbahi, Optimal topology design for dynamic networks, in Proceedings of the 50th IEEE Conference on Decision and Control and European Control Conference (CDCECC), IEEE Press, Piscataway, NJ, 2011, pp. 1280-1285, https://doi.org/10.1109/CDC. 2011.6161026.

[13] S. S. Dey And D. A. Morán R., Some properties of convex hulls of integer points contained in general convex sets, Math. Program., 141 (2013), pp. 507-526.

[14] M. J. Feizollahi, S. Ahmed, And X. A. Sun, Exact augmented Lagrangian duality for mixed integer linear programming, Math. Program., 161 (2017), pp. 365-387.

[15] M. Guzelsoy and T. K. Ralphs, Duality for mixed-integer linear programs, Int. J. Oper. Res. (Taichung), 4 (2007), pp. 118-137.

[16] F. KilinÇ-Karzan And D. E. StefFy, On sublinear inequalities for mixed integer conic programs, Math. Program., 159 (2016), pp. 585-605.

[17] B. Kocuk, S. S. Dey, AND X. A. Sun, New formulation and strong MISOCP relaxations for AC optimal transmission switching problem, IEEE Trans. Power Syst., 32 (2017), pp. 41614170.

[18] R. R. MEYER, On the existence of optimal solutions to integer and mixed-integer programming problems, Math. Program., 7 (1974), pp. 223-235.

[19] D. A. Morán R. And S. S. Dey, On maximal S-free convex sets, SIAM J. Discrete Math., 25 (2011), pp. 379-393.

[20] D. A. Morán R., S. S. Dey, And J. P. Vielma, A strong dual for conic mixed-integer programs, SIAM J. Optim., 22 (2012), pp. 1136-1150.

[21] G. L. Nemhauser and L. A. Wolsey, Integer and Combinatorial Optimization, WileyInterscience, New York, 1988.

[22] M. C. PINAR, Mixed-integer second-order cone programming for lower hedging of American contingent claims in incomplete markets, Optim. Lett., 7 (2013), pp. 63-78, https://doi. org/10.1007/s11590-011-0394-z.

[23] D. A. M. R. AND S. S. DEY, Closedness of integer hulls of simple conic sets, SIAM J. Discrete Math., 30 (2016), pp. 70-99.

[24] G. T. Rockafeller, Convex Analysis, Princeton University Press, Princeton, NJ, 1970.

[25] S. Yildiz and G. CoRnuÉJols, Disjunctive cuts for cross-sections of the second-order cone, Oper. Res. Lett., 43 (2015), pp. 432-437, https://doi.org/10.1016/j.orl.2015.06.001.

[26] S. Sanjeevi, S. Masihabadi, And K. Kianfar, Using cuts for mixed integer knapsack sets to generate cuts for mixed integer polyhedral conic sets, Math. Program., 159 (2016), pp. 571583.

[27] A. Santana And S. S. Dey, Some cut-generating functions for second-order conic sets, Discrete Optim., 24 (2017), pp. 51-65.

[28] A. SchriJver, Theory of Linear and Integer Programming, John Wiley, New York, 1998.

[29] A. Shapiro And A. Nemirovski, Duality of Linear Conic Problems, preprint, School of Industrial and Systems Engineering, Georgia Institute of Technology, Atlanta, GA, 2003.

[30] L. A. Wolsey, Integer programming duality: Price functions and sensitivity analysis, Math. Program., 20 (1981), pp. 173-195. 